\title{
SubRiemannian geometry on the sphere $\mathbb{S}^{3}$ \\ Ovidiu Calin, Der-Chen Chang and Irina Markina 1
}

\begin{abstract}
We discuss the subRiemannian geometry induced by two noncommutative vector fields which are left invariant on the Lie group $\mathbb{S}^{3}$.

Key words: noncommutative Lie group, subRiemannian geodesic, Euler-Lagrange equations MS Classification (2000): 53C17, 53C22, 35H20
\end{abstract}

\section{Introduction}

The study of step 2 subRiemannian manifolds has the Heisenberg group as a prototype. This is a noncommutative Lie group with the base manifold $\mathbb{R}^{3}$ and endowed with a nonintegrable distribution spanned by two of the noncommutative left invariant vector fields. This structure enjoys also the property of being a contact structure or a CR-manifold. The study of the subRiemannian geodesics on the Heisenberg group started with the work of Gaveau [9]. One trend in the literature is to use the geometry of the Heisenberg group to describe the Heisenberg Laplacian and its heat kernel, see Beals, Gaveau, Greiner [1,2,3,4]. Later, this structure led to generalizations of the Heisenberg group as can be seen in Calin, Chang, Greiner $[5,6]$ and Chang, Markina [7]. For more fundamental issues on subRiemannian geometry, see Strichartz [10].

One question is, if there is another 3-dimensional noncommutative Lie group besides the Heisenberg group. The answer is positive and this paper deals with it. The sphere $\mathbb{S}^{3}$ and the group $S U(2)$ have these properties. More important, this is the first compact subRiemannian manifold has been under consideration.

The present paper starts with an introduction to quaternions and then defines the 3dimmensional sphere as the set of quaternions of length one. The quaternion group induces on $\mathbb{S}^{3}$ a structure of noncommutative Lie group. This group is compact and the results obtained in this case are very different than those obtained in the case of the Heisenberg group, which is a noncompact Lie group. Like in the Heisenberg group case, we introduce a nonintegrable distribution on the sphere and a metric on it using two of the noncommutative left invariant vector fields. This way $\mathbb{S}^{3}$ becomes a subRiemannian manifold. It is known that the group $S U(2)$ is isomorphic with the sphere $\mathbb{S}^{3}$ and represents an example of subRiemannian manifold where the elements are matrices. The main issue here is to study the connectivity by horizontal curves and its geodesics on this manifold. In this paper, we are using Lagrangian method to study the connectivity theorem on $\mathbb{S}^{3}$ by horizontal curves with minimal arc-length. We show that for any two points in $\mathbb{S}^{3}$, there exists such a geodesic joining these two points.

\footnotetext{
${ }^{1}$ The first author partially supported by a research grant at Eastern Michigan University. The second author partially supported by a competitive research grant at Georgetown University. The third author is partially supported by a research grant at University of Bergen.
} 
The final version of this paper was in part written while the first two authors visited the National Center for Theoretical Sciences and National Tsing Hua University during June-July of 2006. They would like to express their profound gratitude to Professors Jin Yu and ShuCheng Chang for their invitation and for the warm hospitality extended to them during their stay in Taiwan.

\section{The Quaternions Group Q}

William R. Hamilton introduced quaternions in 1873. They are obtained as a non-commutative extension of the complex numbers. Let $\mathbf{Q}$ be the set of matrices $\left(\begin{array}{cc}\alpha & \beta \\ -\bar{\beta} & \bar{\alpha}\end{array}\right)$ with complex number entries $\alpha, \beta \in \mathbb{C}$. The set $\mathbf{Q}$ becomes a group with the usual matrix multiplication law, called the quaternions group. The identity element is $\mathrm{I}=\left(\begin{array}{ll}1 & 0 \\ 0 & 1\end{array}\right)$ and the inverse of $q=\left(\begin{array}{cc}\alpha & \beta \\ -\bar{\beta} & \bar{\alpha}\end{array}\right)$ is $q^{-1}=\frac{1}{\Delta}\left(\begin{array}{cc}\bar{\alpha} & -\beta \\ \beta & \alpha\end{array}\right)$, where $\Delta=|\alpha|^{2}+|\beta|^{2}$. Let

$$
\mathbf{i}=\left(\begin{array}{cc}
i & 0 \\
0 & -i
\end{array}\right), \quad \mathbf{j}=\left(\begin{array}{cc}
0 & 1 \\
-1 & 0
\end{array}\right), \quad \mathbf{k}=\left(\begin{array}{ll}
0 & i \\
i & 0
\end{array}\right) .
$$

Then

$$
\begin{aligned}
\mathbf{i}^{2}=\mathbf{j}^{2}=\mathbf{k}^{2}=-\mathrm{I}, & \mathbf{i j}=-\mathbf{j} \mathbf{i}=\mathbf{k} \\
\mathbf{j} \mathbf{k}=-\mathbf{k} \mathbf{j}=\mathbf{i}, & \mathbf{k} \mathbf{i}=-\mathbf{i} \mathbf{k}=\mathbf{j}
\end{aligned}
$$

Any real number $a \in \mathbb{R}$ can be identified with $a \mathbf{i}$ by the one to one homomorphism $\varphi: \mathbb{R} \rightarrow \mathbf{Q}$, $\varphi(a)=\left(\begin{array}{cc}a & 0 \\ 0 & -a\end{array}\right)$. If $\alpha=a_{0}+a_{1} \mathbf{i}, \beta=b_{0}+b_{1} \mathbf{i} \in \mathbb{C}$, then an elementary computation yields

$$
q=\left(\begin{array}{cc}
\alpha & \beta \\
-\bar{\beta} & \bar{\alpha}
\end{array}\right)=a_{0} \mathrm{I}+a_{1} \mathbf{i}+b_{0} \mathbf{j}+b_{1} \mathbf{k}
$$

The set $\{\mathrm{I}, \mathbf{i}, \mathbf{j}, \mathbf{k}\}$ is a basis of $\mathbf{Q}$. Let $q=a \mathrm{I}+b \mathbf{i}+c \mathbf{j}+d \mathbf{k}$ and $q^{\prime}=a^{\prime} \mathrm{I}+b^{\prime} \mathbf{i}+c^{\prime} \mathbf{j}+d^{\prime} \mathbf{k}$ be two quaternions.

A straightforward computation shows

$$
\begin{aligned}
q q^{\prime}= & \left(a a^{\prime}-b b^{\prime}-c c^{\prime}-d d^{\prime}\right) \mathrm{I}+\left(a b^{\prime}+b a^{\prime}+c d^{\prime}-d c^{\prime}\right) \mathbf{i} \\
& +\left(a c^{\prime}+c a^{\prime}+d b^{\prime}-b d^{\prime}\right) \mathbf{j}+\left(a d^{\prime}+d a^{\prime}+b c^{\prime}-c b^{\prime}\right) \mathbf{k}
\end{aligned}
$$

The conjugate of $q=a \mathrm{I}+b \mathbf{i}+c \mathbf{j}+d \mathbf{k}$ is $\bar{q}=a \mathrm{I}-b \mathbf{i}-c \mathbf{j}-d \mathbf{k}$. A simple computation shows that $q \bar{q}=a^{2}+b^{2}+c^{2}+d^{2}=\Delta, q^{-1}=\frac{1}{\Delta} \bar{q}$. The modulus of $q$ is $|q|=\sqrt{\Delta}$. Since $\mathbf{i}, \mathbf{j}, \mathbf{k}$ do not commute, the multiplication of quaternions is noncommutative. 


\section{$3 \quad \mathbb{S}^{3}$ as a noncommutative Lie group}

Consider $\mathbb{S}^{3}=\{q \in \mathbf{Q} ;|q|=1\}$. For any $q_{1}, q_{2} \in \mathbb{S}^{3},\left|q_{1}^{-1} q_{2}\right|=\frac{\left|q_{2}\right|}{\left|q_{1}\right|}=1$, i.e. $\mathbb{S}^{3}$ is a subgroup of $\mathbf{Q}$. The law group on $\mathbb{S}^{3}$ is

$$
\begin{aligned}
& (a, b, c, d) *\left(a^{\prime}, b^{\prime}, c^{\prime}, d^{\prime}\right) \\
= & \left(a a^{\prime}-b b^{\prime}-c c^{\prime}-d d^{\prime}, a b^{\prime}+b a^{\prime}+c d^{\prime}-d c^{\prime}, a c^{\prime}+c a^{\prime}+d b^{\prime}-b d^{\prime}, a d^{\prime}+d a^{\prime}+b c^{\prime}-c b^{\prime}\right),
\end{aligned}
$$

with the identity element $(1,0,0,0)$ and the inverse $(a, b, c, d)^{-1}=(a,-b,-c,-d)$. In this way $\left(\mathbb{S}^{3}, *\right)$ becomes a compact, noncommutative Lie group.

We shall work out the left invariant vector fields with respect to the left translation $L_{q}$ : $\mathbb{S}^{3} \rightarrow \mathbb{S}^{3}, L_{q} h=q * h$. These are vector fields on $\mathbb{S}^{3}$ such that

$$
d L_{q}\left(X_{h}\right)=X_{q * h}, \quad \forall q, h \in \mathbb{S}^{3} .
$$

The set of all left invariant vector fields on $\mathbb{S}^{3}$

$$
\mathcal{L}\left(\mathbb{S}^{3}\right)=\left\{X ; d L_{q} X=X, \forall q \in \mathbb{S}^{3}\right\}
$$

is called the Lie algebra of $\mathbb{S}^{3} . \mathcal{L}\left(\mathbb{S}^{3}\right)$ is a subalgebra of $\mathcal{L}(Q)$, i.e. it is a subset closed under the Lie bracket $[$,$] .$

In order to find a basis for $\mathcal{L}\left(\mathbb{S}^{3}\right)$, we shall find first a basis for $\mathcal{L}(\mathbf{Q})$. Let $X$ be a left invariant vector field on $\mathbf{Q}$. Then $X_{q}=\left(d L_{q}\right) X_{e}$, where $e=(1,0,0,0)$ is the identity of $\mathbf{Q}$. If write

$$
X_{q}=\sum_{i=1}^{4} X_{q}^{i} \frac{\partial}{\partial x_{i}}
$$

the components are

$$
X_{q}^{i}=\left(d L_{q}\right) X_{e}\left(x_{i}\right)=X_{e}\left(x_{i} \circ L_{q}\right), \quad i=1, \ldots, 4,
$$

where $x_{i}$ is the $i$-th coordinate.

Next we shall compute $x_{i} \circ L_{q}, i=1,2,3,4$. Let $q=(a, b, c, d)$ and $q^{\prime}=\left(a^{\prime}, b^{\prime}, c^{\prime}, d^{\prime}\right)$. Then

$$
\left(x_{1} \circ L_{q}\right)\left(q^{\prime}\right)=x_{1}\left(L_{q} q^{\prime}\right)=a a^{\prime}-b b^{\prime}-c c^{\prime}-d d^{\prime} .
$$

Using

$$
\begin{aligned}
& a=x_{1}(q), \quad a^{\prime}=x_{1}\left(q^{\prime}\right), \quad b=x_{2}(q), \quad b^{\prime}=x_{2}\left(q^{\prime}\right), \\
& c=x_{3}(q), \quad c^{\prime}=x_{3}\left(q^{\prime}\right), \quad d=x_{4}(q), \quad d^{\prime}=x_{4}\left(q^{\prime}\right),
\end{aligned}
$$


equation (3.2) becomes

$$
\left(x_{1} \circ L_{q}\right)\left(q^{\prime}\right)=x_{1}(q) x_{1}\left(q^{\prime}\right)-x_{2}(q) x_{2}\left(q^{\prime}\right)-x_{3}(q) x_{3}\left(q^{\prime}\right)-x_{4}(q) x_{4}\left(q^{\prime}\right) .
$$

Dropping the argument $q^{\prime}$, yields

$$
x_{1} \circ L_{q}=x_{1}(q) x_{1}-x_{2}(q) x_{2}-x_{3}(q) x_{3}-x_{4}(q) x_{4} .
$$

By a similar computation we find

$$
\begin{aligned}
& x_{2} \circ L_{q}=x_{1}(q) x_{2}+x_{2}(q) x_{1}+x_{3}(q) x_{4}-x_{4}(q) x_{3}, \\
& x_{3} \circ L_{q}=x_{1}(q) x_{3}+x_{3}(q) x_{1}+x_{4}(q) x_{2}-x_{2}(q) x_{4}, \\
& x_{4} \circ L_{q}=x_{1}(q) x_{4}+x_{4}(q) x_{1}+x_{2}(q) x_{3}-x_{3}(q) x_{2} .
\end{aligned}
$$

The first component becomes

$$
\begin{aligned}
X_{q}^{1} & =X_{e}\left(x_{1} \circ L_{q}\right) \\
& =x_{1}(q) X_{e}\left(x_{1}\right)-x_{2}(q) X_{e}\left(x_{2}\right)-x_{3}(q) X_{e}\left(x_{3}\right)-x_{4}(q) X_{e}\left(x_{4}\right) \\
& =x_{1}(q) X_{e}^{1}-x_{2}(q) X_{e}^{2}-x_{3}(q) X_{e}^{3}-x_{4}(q) X_{e}^{4} .
\end{aligned}
$$

Dropping $q$ and denoting $\xi^{i}=X_{e}^{i}, i=1,2,3,4$, the previous relation yields

$$
X^{1}=x_{1} \xi^{1}-x_{2} \xi^{2}-x_{3} \xi^{3}-x_{4} \xi^{4} .
$$

In a similar way we obtain

$$
\begin{aligned}
X^{2} & =x_{1} \xi^{2}+x_{2} \xi^{1}+x_{3} \xi^{4}-x_{4} \xi^{3} \\
X^{3} & =x_{1} \xi^{3}+x_{3} \xi^{1}+x_{4} \xi^{2}-x_{2} \xi^{4} \\
X^{4} & =x_{1} \xi^{4}+x_{4} \xi^{1}+x_{2} \xi^{3}-x_{3} \xi^{2} .
\end{aligned}
$$

Left invariant vector fields $Z$ can be written as

$$
\begin{aligned}
Z=\sum_{i=1}^{4} Z^{i} \frac{\partial}{\partial x_{i}}= & \left(x_{1} \xi^{1}-x_{2} \xi^{2}-x_{3} \xi^{3}-x_{4} \xi^{4}\right) \frac{\partial}{\partial x_{1}} \\
& +\left(x_{1} \xi^{2}+x_{2} \xi^{1}+x_{3} \xi^{4}-x_{4} \xi^{3}\right) \frac{\partial}{\partial x_{2}} \\
& +\left(x_{1} \xi^{3}+x_{3} \xi^{1}+x_{4} \xi^{2}-x_{2} \xi^{4}\right) \frac{\partial}{\partial x_{3}} \\
& +\left(x_{1} \xi^{4}+x_{4} \xi^{1}+x_{2} \xi^{3}-x_{3} \xi^{2}\right) \frac{\partial}{\partial x_{4}}
\end{aligned}
$$


Reordering, yields

$$
\begin{aligned}
Z= & \xi^{1}\left[x_{1} \frac{\partial}{\partial x_{1}}+x_{2} \frac{\partial}{\partial x_{2}}+x_{3} \frac{\partial}{\partial x_{3}}+x_{4} \frac{\partial}{\partial x_{4}}\right] \\
& +\xi^{2}\left[-x_{2} \frac{\partial}{\partial x_{1}}+x_{1} \frac{\partial}{\partial x_{2}}+x_{4} \frac{\partial}{\partial x_{3}}-x_{3} \frac{\partial}{\partial x_{4}}\right] \\
& +\xi^{3}\left[-x_{3} \frac{\partial}{\partial x_{1}}-x_{4} \frac{\partial}{\partial x_{2}}+x_{1} \frac{\partial}{\partial x_{3}}+x_{2} \frac{\partial}{\partial x_{4}}\right] \\
& +\xi^{4}\left[-x_{4} \frac{\partial}{\partial x_{1}}+x_{3} \frac{\partial}{\partial x_{2}}-x_{2} \frac{\partial}{\partial x_{3}}+x_{1} \frac{\partial}{\partial x_{4}}\right] \\
= & \xi^{1} N-\xi^{2} X-\xi^{3} T-\xi^{4} Y,
\end{aligned}
$$

where

$$
N=\sum_{i=1}^{4} x_{i} \frac{\partial}{\partial x_{i}}
$$

is the normal vector field to $\mathbb{S}^{3}$, and

$$
\begin{aligned}
X & =x_{2} \frac{\partial}{\partial x_{1}}-x_{1} \frac{\partial}{\partial x_{2}}-x_{4} \frac{\partial}{\partial x_{3}}+x_{3} \frac{\partial}{\partial x_{4}}, \\
Y & =x_{4} \frac{\partial}{\partial x_{1}}-x_{3} \frac{\partial}{\partial x_{2}}+x_{2} \frac{\partial}{\partial x_{3}}-x_{1} \frac{\partial}{\partial x_{4}}, \\
T & =x_{3} \frac{\partial}{\partial x_{1}}+x_{4} \frac{\partial}{\partial x_{2}}-x_{1} \frac{\partial}{\partial x_{3}}-x_{2} \frac{\partial}{\partial x_{4}} .
\end{aligned}
$$

Using the $4 \times 4$-matrix representations:

$$
I_{1}=\left[\begin{array}{rrrr}
0 & 1 & 0 & 0 \\
-1 & 0 & 0 & 0 \\
0 & 0 & 0 & -1 \\
0 & 0 & 1 & 0
\end{array}\right], \quad I_{3}=\left[\begin{array}{rrrr}
0 & 0 & 1 & 0 \\
0 & 0 & 0 & 1 \\
-1 & 0 & 0 & 0 \\
0 & -1 & 0 & 0
\end{array}\right], \quad I_{2}=\left[\begin{array}{rrrr}
0 & 0 & 0 & 1 \\
0 & 0 & -1 & 0 \\
0 & 1 & 0 & 0 \\
-1 & 0 & 0 & 0
\end{array}\right]
$$

and the standard inner product $\langle$,$\rangle on \mathbb{R}^{4}$, we also can write

$$
N=\left\langle x U, \nabla_{x}\right\rangle, \quad X=-\left\langle x I_{1}, \nabla_{x}\right\rangle, \quad Y=-\left\langle x I_{3}, \nabla_{x}\right\rangle, \quad T=-\left\langle x I_{2}, \nabla_{x}\right\rangle,
$$

where $U$ is the $4 \times 4$ identity matrix.

It is easy to see that $\{N, X, Y, T\}$ is a basis for $\mathcal{L}(\mathbf{Q})$. In order to find a basis for $\mathcal{L}\left(\mathbb{S}^{3}\right)$ we shall consider the vector fields which are tangent to $\mathbb{S}^{3}$. Since a computation provides

$$
\langle N, X\rangle=\langle N, Y\rangle=\langle N, T\rangle=0,
$$

it follows that $X, Y, T$ are tangent to $\mathbb{S}^{3}$. Hence any left invariant vector field on $\left(\mathbb{S}^{3}, *\right)$ is a linear combination of $X, Y$ and $T$. Since the matrix of coefficients

$$
\left(\begin{array}{rrrr}
x_{2} & -x_{1} & -x_{4} & x_{3} \\
x_{4} & -x_{3} & x_{2} & -x_{1} \\
x_{3} & x_{4} & -x_{1} & -x_{2}
\end{array}\right)
$$


has rank 3 at every point, it follows that $X, Y$ and $T$ are linear independent and hence form a basis of $L\left(\mathbb{S}^{3}\right)$. It worth nothing that the above rank property is not true without the constraint $x_{1}^{2}+x_{2}^{2}+x_{3}^{3}+x_{4}^{2}=1$.

We also note that $\{X, Y, T, N\}$ form an orthonormal system with respect to the usual inner product of $\mathbb{R}^{4}$. Then $\mathcal{L}(\mathbf{Q})=\mathcal{L}\left(\mathbb{S}^{3}\right) \bigoplus \mathbb{R} N$.

\subsection{The horizontal distribution}

Let $\mathcal{H}=\operatorname{span}\{X, Y\}$ be the distribution generated by the vector fields $X$ and $Y$. Since $[Y, X]=2 T \notin \mathcal{H}$ it follows that $\mathcal{H}$ is not involutive. We can write $\mathcal{L}\left(\mathbb{S}^{3}\right)=\mathcal{H} \oplus \mathbb{R} T$. The distribution $\mathcal{H}$ will be called the horizontal distribution. Any curve on the sphere which has the velocity vector contained in the distribution $\mathcal{H}$ will be called a horizontal curve.

Remark 3.1 Notice that this situation differs from the Heisenberg group since the choice of the horizontal distribution is not unique. Because of $[X, T]=-2 Y$ and $[Y, T]=2 X$ we could chose $\mathcal{H}=\operatorname{span}\{X, T\}$ or $\mathcal{H}=\operatorname{span}\{Y, T\}$. The geometries defining by different horizontal distributions are cyclically symmetric, so we restrict out attention to the $\mathcal{H}=\operatorname{span}\{X, Y\}$.

The following result deals with a characterization of horizontal curves. It will make sense for later reasons to rename the variables $y_{1}=x_{3}, y_{2}=x_{4}$.

Proposition 3.2 Let $\gamma(s)=\left(x_{1}(s), x_{2}(s), y_{1}(s), y_{2}(s)\right)$ be a curve on $\mathbb{S}^{3}$. The curve $\gamma$ is horizontal if and only if

$$
\langle\dot{x}, y\rangle=\langle x, \dot{y}\rangle .
$$

Proof: Since $\{X, Y, T\}$ span the tangent space of the sphere $\mathbb{S}^{3}$ we have

$$
\dot{\gamma}=a X+b Y+c T .
$$

Using that $\{X, Y, T\}$ is an orthonormal system on $\mathbb{S}^{3}$, we have

$$
\begin{aligned}
c & =\langle\dot{\gamma}, T\rangle \\
& =\dot{x}_{1} y_{1}+\dot{x}_{2} y_{2}-\dot{y}_{1} x_{1}-\dot{y}_{2} x_{2} \\
& =\langle\dot{x}, y\rangle-\langle x, \dot{y}\rangle .
\end{aligned}
$$

The curve $\gamma$ is horizontal if and only if $c=0$ i.e. $\langle\dot{x}, y\rangle=\langle x, \dot{y}\rangle$.

Hence a horizontal curve $\gamma=\left(x_{1}, x_{2}, y_{1}, y_{2}\right):(0, \tau) \rightarrow \mathbb{R}^{3}$ has the velocity $\dot{\gamma}=a X+b Y$ where

$$
\begin{aligned}
a & =\langle\dot{\gamma}, X\rangle=\dot{x}_{1} x_{2}-\dot{x}_{2} x_{1}-\dot{y}_{1} y_{2}+\dot{y}_{2} y_{1} \\
b & =\langle\dot{\gamma}, Y\rangle=\dot{x}_{1} y_{2}-\dot{x}_{2} y_{1}+\dot{y}_{1} x_{2}-\dot{y}_{2} x_{1}
\end{aligned}
$$


If we consider a metric on $\mathcal{H}$ such that $\{Y, X\}$ are orthonormal vector fields, then the length of the curve $\gamma$ is

$$
\ell(\gamma)=\int_{0}^{T} \sqrt{a(s)^{2}+b(s)^{2}} d s
$$

The horizontal curves minimizing length will be treated in a next section.

In the rest of this section we shall make a few considerations regarding the horizontal distribution given as the kernel of a one-form.

Consider the following one-form on $\mathbb{R}^{4}$

$$
\omega=x_{1} d y_{1}-y_{1} d x_{1}+x_{2} d y_{2}-y_{2} d x_{2}=x d y-y d x .
$$

One can easily check that

$$
\begin{aligned}
\omega(X) & =-x_{1} y_{2}-y_{1} x_{2}+x_{2} y_{1}+x_{1} y_{2}=0 \\
\omega(Y) & =x_{1} x_{2}-y_{1} y_{2}-x_{1} x_{2}+y_{1} y_{2}=0 \\
\omega(T) & =-x_{1}^{2}-y_{1}^{2}-x_{2}^{2}-y_{2}^{2}=-1 \neq 0 \\
\omega(N) & =x_{1} y_{1}-y_{1} x_{1}+x_{2} y_{2}-y_{2} x_{2}=0
\end{aligned}
$$

where

$$
N=x_{1} \partial_{x_{1}}+x_{2} \partial_{x_{2}}+y_{1} \partial_{y_{1}}+y_{2} \partial_{y_{2}}
$$

is the unit normal to the sphere $\mathbb{S}^{3}$. Hence $\operatorname{ker} \omega=\operatorname{span}\{X, Y, N\}$ and the horizontal distribution can be written as

$$
\mathbb{S}^{3} \ni p \rightarrow \mathcal{H}_{p}=\operatorname{ker} \omega \cap T_{p} \mathbb{S}^{3} .
$$

Hence a vector $\nu=(v, w)=\left(v_{1}, v_{2}, w_{1}, w_{2}\right) \in \mathcal{H}$ if and only if

$$
\begin{aligned}
\langle x, v\rangle+\langle y, w\rangle & =0(\Longleftrightarrow \nu \perp N) \\
\langle v, y\rangle-\langle x, w\rangle & =0(\Longleftrightarrow \omega(\nu)=0) \\
|x|^{2}+|y|^{2} & =1\left(\Longleftrightarrow(x, y) \in \mathbb{S}^{3}\right) .
\end{aligned}
$$

\section{Connectivity theorem on $\mathbb{S}^{3}$}

The present section deals with the global connectivity property by horizontal curves. Even if a result of Chow [8] states the connectivity by piece-wise horizontal curves, here we shall prove a globally smooth version of it. More precisely, the main result of this section states that given two points on $\mathbb{S}^{3}$, there is a horizontal smooth curve joining them.

Before proceeding to the proof, we need to introduce some terminology and notations. This deals with the parametrization of $\mathbb{S}^{3}$ in terms of the Euler angles, which due to spherical symmetry are more suitable than the cartesian coordinates. 
Consider $\varphi, \psi, \theta$ to be the Euler's angles and let

$$
\alpha=\frac{\varphi+\psi}{2}, \quad \beta=\frac{\varphi-\psi}{2} .
$$

The sphere $\mathbb{S}^{3}$ can be parametrized as

$$
\begin{aligned}
& x_{1}=\cos \frac{\varphi+\psi}{2} \cos \frac{\theta}{2}=\cos \alpha \cos \frac{\theta}{2} \\
& x_{2}=\sin \frac{\varphi+\psi}{2} \cos \frac{\theta}{2}=\sin \alpha \cos \frac{\theta}{2} \\
& y_{1}=\cos \frac{\varphi-\psi}{2} \sin \frac{\theta}{2}=\cos \beta \sin \frac{\theta}{2} \\
& y_{2}=\sin \frac{\varphi-\psi}{2} \sin \frac{\theta}{2}=\sin \beta \sin \frac{\theta}{2}
\end{aligned}
$$

with $\quad 0 \leq \theta \leq \pi, \quad-\pi \leq \alpha \leq \pi$. In the following we shall write the restriction of the one-form

$$
\omega=x_{1} d y_{1}-y_{1} d x_{1}+x_{2} d y_{2}-y_{2} d x_{2}
$$

to $\mathbb{S}^{3}$ using Euler's angles. Since

$$
\begin{aligned}
d x_{1}= & -\sin \alpha \cos \frac{\theta}{2} d \alpha-\frac{1}{2} \cos \alpha \sin \frac{\theta}{2} d \theta \\
d x_{2}= & \cos \alpha \cos \frac{\theta}{2} d \alpha-\frac{1}{2} \sin \alpha \sin \frac{\theta}{2} d \theta \\
d y_{1}= & -\sin \beta \sin \frac{\theta}{2} d \beta+\frac{1}{2} \cos \beta \cos \frac{\theta}{2} d \theta \\
d y_{2}= & \cos \beta \sin \frac{\theta}{2} d \beta+\frac{1}{2} \sin \beta \cos \frac{\theta}{2} d \theta,
\end{aligned}
$$

we obtain

$$
\begin{aligned}
\omega= & x_{1} d y_{1}-y_{1} d x_{1}+x_{2} d y_{2}-y_{2} d x_{2} \\
= & \left(\cos \beta \sin \alpha \sin \frac{\theta}{2} \cos \frac{\theta}{2}-\sin \beta \cos \alpha \sin \frac{\theta}{2} \cos \frac{\theta}{2}\right) d \alpha \\
& +\left(-\cos \alpha \cos \frac{\theta}{2} \sin \beta \sin \frac{\theta}{2}+\sin \alpha \cos \frac{\theta}{2} \sin \frac{\theta}{2} \cos \beta\right) d \beta \\
& +\frac{1}{2}\left(\cos \alpha \cos \frac{\theta}{2} \cos \beta \cos \frac{\theta}{2}+\cos \alpha \cos \beta \sin ^{2} \frac{\theta}{2}+\sin \alpha \sin \beta \cos ^{2} \frac{\theta}{2}+\sin \beta \sin \alpha \sin ^{2} \frac{\theta}{2}\right) d \theta \\
= & \sin \frac{\theta}{2} \cos \frac{\theta}{2} \sin (\alpha-\beta) d \alpha+\sin \frac{\theta}{2} \cos \frac{\theta}{2} \sin (\alpha-\beta) d \beta+\frac{1}{2} \cos (\beta-\alpha) d \theta \\
= & \frac{1}{2} \sin \theta \sin \psi(d \alpha+d \beta)+\frac{1}{2} \cos \psi d \theta \\
= & \frac{1}{2}(\sin \theta \sin \psi d \varphi+\cos \psi d \theta) .
\end{aligned}
$$


Hence

$$
\omega_{\mid \mathbb{S}^{3}}=\frac{1}{2}(\sin \theta \sin \psi d \varphi+\cos \psi d \theta) .
$$

The constraint $\sin \theta \sin \psi d \varphi+\cos \psi d \theta=0$ is nonholonomic since

$$
2 d \omega=\cos \theta \sin \psi d \theta \wedge d \varphi+\sin \theta \cos \psi d \psi \wedge d \varphi-\sin \psi d \psi \wedge d \theta \neq 0 .
$$

We finally arrived at the following characterization of the horizontal curves using Euler's angles.

Lemma 4.1 Let $c(s)=(\varphi(s), \psi(s), \theta(s))$ be a curve on $\mathbb{S}^{3}$. The curve $c$ is horizontal if and only if $\omega(\dot{c})=0$, i.e.

$$
\sin \theta \sin \psi \dot{\varphi}+\cos \psi \dot{\theta}=0 .
$$

Before getting into the proof of the connectivity theorem, which is the main result of this section, a couple of lemmas are needed. The first one is a standard Calculus exercise.

Lemma 4.2 Given the numbers $\alpha, \beta, \gamma \in \mathbb{R}$, there is a smooth function $f:[0,1] \rightarrow \mathbb{R}$ such that

$$
f(0)=0, \quad f(1)=\alpha, \quad f^{\prime}(0)=\beta, \quad f^{\prime}(1)=\gamma .
$$

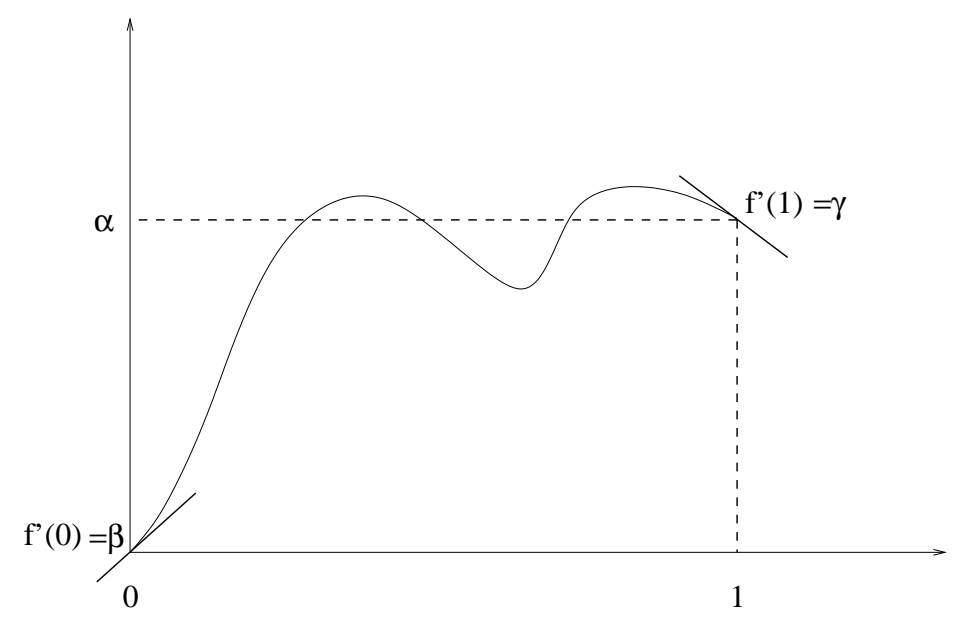

Lemma 4.3 Given $q_{0}, q_{1}, I \in \mathbb{R}$, there is a function $q:[0,1] \rightarrow \mathbb{R}$ such that

$$
q(0)=q_{0}, \quad q(1)=q_{1}, \quad \int_{0}^{1} q(u) d u=I .
$$


Proof: Applying Lemma 4.2, there is a function $f:[0,1] \rightarrow \mathbb{R}$ such that

$$
f(0)=0, \quad f(1)=I, \quad f^{\prime}(0)=q_{0}, \quad f^{\prime}(1)=q_{1} .
$$

Let $q(s)=f^{\prime}(s)$. Then $f(s)=\int_{0}^{s} q(u) d u$ and hence $I=f(1)=\int_{0}^{1} q(u) d u$ and $q(0)=f^{\prime}(0)=$ $q_{0}$ and $q(1)=f^{\prime}(1)=q_{1}$.

The next result is the theorem of connectivity announced at the beginning of this section.

Theorem 4.4 Given two points $P, Q \in \mathbb{S}^{3}$, there is a smooth horizontal curve joining $P$ and $Q$.

Proof: Let $P\left(\varphi_{0}, \psi_{0}, \theta_{0}\right)$ and $Q\left(\varphi_{1}, \psi_{1}, \theta_{1}\right)$ be the coordinates in the Eulerian angles. We shall find a horizontal curve $c(s)=(\varphi(s), \psi(s), \theta(s))$ with $c(0)=P$ and $c(1)=Q$. This is equivalent with finding functions $\varphi(s), \psi(s), \theta(s)$ such that

$$
\sin \theta(s) \sin \psi(s) \dot{\varphi}(s)+\cos \psi(s) \dot{\theta}(s)=0,
$$

which satisfy the boundary conditions

$$
\begin{array}{lll}
\varphi(0)=\varphi_{0}, & \psi(0)=\psi_{0}, & \theta(0)=\theta_{0}, \\
\varphi(1)=\varphi_{1}, & \psi(1)=\psi_{1}, & \theta(1)=\theta_{1} .
\end{array}
$$

Assuming $\cos \psi(s) \neq 0$, (4.8) can be written as

$$
\sin \theta(s) \tan \psi(s) \dot{\varphi}(s)+\dot{\theta}(s)=0 .
$$

Let

$$
\theta(s)=\arcsin p(s), \quad \psi(s)=\arctan q(s), \quad \varphi(s)=\varphi_{0}+s\left(\varphi_{1}-\varphi_{0}\right)
$$

for some functions $p(s), q(s)$ which will be determined later. Let $k=\varphi_{1}-\varphi_{0}$. Then (4.9) yields

$$
p(s) q(s) k+\frac{p^{\prime}(s)}{\sqrt{1-p^{2}(s)}}=0 .
$$

Separating and solving for $p(s)$ yields

$$
\frac{d p}{p \sqrt{1-p^{2}}}=-k q(s) d s
$$

and integrating,

$$
\begin{aligned}
-\tanh ^{-1} \frac{1}{\sqrt{1-p^{2}}} & =-k\left(\int_{0}^{s} q(u) d u+C_{1}\right) \Longleftrightarrow \\
\frac{1}{\sqrt{1-p^{2}(s)}} & =\tanh \left[k\left(\int_{0}^{s} q(u) d u+C_{1}\right)\right] .
\end{aligned}
$$


The constant $C_{1}$ and the integral $\int_{0}^{1} q(u) d u$ can be determined from the boundary conditions. We obtain

$$
\begin{gathered}
\frac{1}{\sqrt{1-p^{2}(0)}}=\tanh \left(k C_{1}\right) \quad \Longrightarrow C_{1}=\frac{1}{k} \tanh ^{-1}\left(\frac{1}{\sqrt{1-p^{2}(0)}}\right) \\
\frac{1}{\sqrt{1-p^{2}(1)}}=\tanh \left[k\left(\int_{0}^{1} q(u) d u+C_{1}\right)\right] \Longrightarrow \\
\int_{0}^{1} q(u) d u=\frac{1}{k}\left[\tanh ^{-1} \frac{1}{\sqrt{1-p^{2}(1)}}-\tanh ^{-1} \frac{1}{\sqrt{1-p^{2}(0)}}\right],
\end{gathered}
$$

where $p(0)=\sin \theta(0)=\sin \theta_{0}$ and $p(1)=\sin \theta_{1}$. Hence $q(s)$ has to satisfy

$$
\int_{0}^{1} q(u) d u=\frac{1}{k}\left[\tanh ^{-1} \frac{1}{\left|\cos \theta_{1}\right|}-\tanh ^{-1} \frac{1}{\left|\cos \theta_{0}\right|}\right] .
$$

The boundary conditions also yield

$$
q(0)=\tan \psi_{0}, \quad q(1)=\tan \psi_{1}
$$

Lemma 4.3 provides the existence of a function $q:[0,1] \rightarrow \mathbb{R}$ such that (4.11) and (4.12) are satisfied.

The function $p(s)$ will be defined by the relation

$$
\frac{1}{\sqrt{1-p^{2}(s)}}=\tanh \left[k \int_{0}^{s} q(u) d u+\tanh ^{-1} \frac{1}{\left|\cos \theta_{0}\right|}\right] .
$$

Hence the curve $c(s)=(\varphi(s), \psi(s), \theta(s))=\left(\varphi_{0}+k s, \arctan q(s), \arcsin p(s)\right)$ is the desired horizontal curve joining the points $P$ and $Q$ on $\mathbb{S}^{3}$.

It is known that in the case of the Heisenberg group if $\left(x_{0}, 0\right)$ and $\left(x_{1}, 0\right)$ are two points in the $\{t=0\}$ plane, then the segment joining the points is a horizontal curve joining the points which lies in the $\{t=0\}$ plane. The following theorem is an analog for the sphere $\mathbb{S}^{3}$. The plane will be replaced by the sphere $\mathbb{S}^{2}$.

Theorem 4.5 Given $\left(\varphi_{0}, \varphi_{1}\right)$ and $\left(\theta_{0}, \theta_{1}\right)$, there is a horizontal curve with $\psi=$ constant, which joins the points with Euler coordinates $\left(\varphi_{0}, \psi, \theta_{0}\right)$ and $\left(\varphi_{1}, \psi, \theta_{1}\right)$.

Proof: Let $(\varphi, \psi, \theta)$ be a horizontal curve with $\psi=$ constant. Then equation (4.7) can be written as

$$
-\tan \psi \frac{d \varphi}{d \theta}=\frac{1}{\sin \theta}
$$


Integrating, yields

$$
\begin{aligned}
-\tan \psi \int_{\theta_{0}}^{\theta} \frac{d \varphi}{d \theta} d \theta & =\int_{\theta_{0}}^{\theta} \frac{1}{\sin \theta} d \theta \Longleftrightarrow \\
-\tan \psi\left(\varphi(\theta)-\varphi\left(\theta_{0}\right)\right) & =\ln \tan \frac{\theta}{2}-\ln \tan \frac{\theta_{0}}{2} .
\end{aligned}
$$

For $\theta=\theta_{1}$ we obtain

$$
\psi=\arctan \left(\ln \frac{\tan \frac{\theta_{1}}{2}}{\tan \frac{\theta_{0}}{2}} /\left(\varphi_{0}-\varphi_{1}\right)\right) .
$$

Solving for $\varphi$, 4.13) yields

$$
\varphi(\theta)-\varphi_{0}=-\ln \left(\tan \frac{\theta}{2} / \tan \frac{\theta_{0}}{2}\right) / \tan \psi,
$$

with $\psi$ defined by (4.14). Hence the horizontal curve joining $\left(\varphi_{0}, \psi, \theta_{0}\right)$ and $\left(\varphi_{1}, \psi, \theta_{1}\right)$ is

$$
(\varphi, \psi, \theta)=\left(\varphi_{0}-\ln \left(\tan \frac{\theta}{2} / \tan \frac{\theta_{0}}{2}\right) / \tan \psi, \psi, \theta\right) .
$$

Let $S_{\psi_{0}}=\left\{(\varphi, \psi, \theta) \in \mathbb{S}^{3} ; \psi=\psi_{0}\right\}$ be the subset of $\mathbb{S}^{3}$ with the same Euler angle $\psi$. This corresponds to a 2-dimensional sphere.

Corollary 4.6 Given the points $\left(\varphi_{0}, \psi_{0}, \theta_{0}\right)$ and $\left(\varphi_{1}, \psi_{1}, \theta_{1}\right)$ on $\mathbb{S}^{3}$, with $\psi_{0}=\psi_{1}$, there is a horizontal curve in $S_{\psi_{0}}$ which joins the points.

\section{The subRiemannian geodesics}

Given two points on the sphere $\mathbb{S}^{3}$, we already know that there are horizontal curves joining them. The horizontal curve with the shortest length is called a geodesic. Standard arguments show that an equivalent problem of finding the geodesics is to find the horizontal curves with minimum energy. This means to minimize the action integral

$$
\int_{0}^{T} \frac{1}{2}\left[a^{2}(s)+b^{2}(s)\right] d s
$$

subject to the horizontality constraint $\langle\dot{x}, y\rangle=\langle x, \dot{y}\rangle$. This will be minimizers given by the solutions of the Euler-Lagrange system with Lagrangian

$$
L(x, \dot{x}, y, \dot{y})=\frac{1}{2}\left(a^{2}+b^{2}\right)+\lambda(s)\left(x_{1} \dot{y}_{1}-y_{1} \dot{x}_{1}+x_{2} \dot{y}_{2}-y_{2} \dot{x}_{2}\right),
$$

where $a$ and $b$ depend on $x, \dot{x}, y, \dot{y}$, see formulas (3.4)-(3.5). The function $\lambda(s)$ is a Lagrange multiplier function. 


\subsection{The case $\lambda(s)=0$.}

The curve which minimizes the energy in this case satisfies the Euler-Lagrange system with the Lagrangian $L(x, y, \dot{x}, \dot{y})=\frac{1}{2} a^{2}+\frac{1}{2} b^{2}$. We have

$$
\begin{aligned}
\frac{\partial L}{\partial \dot{x}_{1}} & =a x_{2}+b y_{2}, \quad \frac{\partial L}{\partial x_{1}}=-a \dot{x}_{2}-b \dot{y}_{2} \\
\frac{\partial L}{\partial \dot{x}_{2}} & =-a x_{1}-b y_{1}, \quad \frac{\partial L}{\partial x_{2}}=a \dot{x}_{1}+b \dot{y}_{1} \\
\frac{\partial L}{\partial \dot{y}_{1}} & =-a y_{2}+b x_{2}, \quad \frac{\partial L}{\partial y_{1}}=a \dot{y}_{2}-b \dot{x}_{2} \\
\frac{\partial L}{\partial \dot{y}_{2}} & =a y_{1}-b x_{1}, \quad \frac{\partial L}{\partial y_{2}}=-a \dot{y}_{1}+b \dot{x}_{1} .
\end{aligned}
$$

Then the Euler-Lagrange system

$$
\begin{aligned}
\frac{d}{d s} \frac{\partial L}{\partial \dot{x}_{1}} & =\frac{\partial L}{\partial x_{1}}, & \frac{d}{d s} \frac{\partial L}{\partial \dot{x}_{2}} & =\frac{\partial L}{\partial x_{2}}, \\
\frac{d}{d s} \frac{\partial L}{\partial \dot{y}_{1}} & =\frac{\partial L}{\partial y_{1}}, & \frac{d}{d s} \frac{\partial L}{\partial \dot{y}_{2}} & =\frac{\partial L}{\partial y_{2}}
\end{aligned}
$$

becomes

$$
\begin{array}{ll}
\dot{a} x_{2}+\dot{b} y_{2}=-2\left(a \dot{x}_{2}+b \dot{y}_{2}\right), & \dot{a} x_{1}+\dot{b} y_{1}=-2\left(a \dot{x}_{1}+b \dot{y}_{1}\right), \\
\dot{a} y_{2}-\dot{b} x_{2}=-2\left(a \dot{y}_{2}-b \dot{x}_{2}\right), & \dot{a} y_{1}-\dot{b} x_{1}=-2\left(a \dot{y}_{1}-b \dot{x}_{1}\right) .
\end{array}
$$

Multiplying the first equation by $x_{2}$, the second by $x_{1}$, the third by $y_{2}$ and the fourth by $y_{1}$, adding yields

$$
\begin{aligned}
& \dot{a}\left(x_{1}^{2}+x_{2}^{2}+y_{2}^{2}+y_{1}^{2}\right)+\dot{b}\left(y_{2} x_{2}+y_{1} x_{1}-x_{2} y_{2}-x_{1} y_{1}\right) \\
= & -2\left[a\left(x_{2} \dot{x}_{2}+x_{1} \dot{x}_{1}+y_{2} \dot{y}_{2}+y_{1} \dot{y}_{1}\right)+b\left(x_{2} \dot{y}_{2}+x_{1} \dot{y}_{1}-y_{2} \dot{x}_{2}-y_{1} \dot{x}_{1}\right)\right] \\
\Longleftrightarrow \dot{a}= & -2 b\left(y_{2} \dot{x}_{2}+y_{1} \dot{x}_{1}-x_{2} \dot{y}_{2}-x_{1} \dot{y}_{1}\right) \\
\Longleftrightarrow \dot{a}= & 0,
\end{aligned}
$$

where we have used the horizontality condition (see Proposition 3.2)

$$
y_{2} \dot{x}_{2}+y_{1} \dot{x}_{1}-x_{2} \dot{y}_{2}-x_{1} \dot{y}_{1}=0,
$$

the constraint

and its derivative

$$
x_{1}^{2}+x_{2}^{2}+y_{1}^{2}+y_{2}^{2}=1
$$

$$
x_{1} \dot{x}_{1}+x_{2} \dot{x}_{2}+y_{1} \dot{y}_{1}+y_{2} \dot{y}_{2}=0 .
$$

In a similar way, multiplying the first equation by $y_{2}$, the second by $y_{1}$, the third by $-x_{2}$, and the fourth by $-x_{1}$, adding, yields $\dot{b}=0$. We arrived at the following result, which provides four constants of motion (three angles and the energy). 
Theorem 5.1 Given two points $P, Q \in \mathbb{S}^{3}$, let $\gamma$ be a length minimizing curve among all the horizontal curves which join $P$ and $Q$. Then

1) $|\dot{\gamma}(s)|$ is constant along the curve.

2) The angles between the velocity $\dot{\gamma}$ and the vector fields $X, Y$ and $T$ are constant along the curve.

Proof:

1) From $\dot{a}=\dot{b}=0$ it follows that $a(s)=$ constant and $b(s)=$ constant and hence $|\dot{\gamma}(s)|=$ $\sqrt{a(s)^{2}+b(s)^{2}}=$ constant.

2) Using 1), we have

$$
\begin{gathered}
\cos \widehat{\dot{\gamma}, X}=\frac{\langle\dot{\gamma}, X\rangle}{|\dot{\gamma}||X|}=\frac{a(s)}{\sqrt{a(s)^{2}+b(s)^{2}}}=\text { constant } \\
\cos \widehat{\dot{\gamma}, Y}=\frac{\langle\dot{\gamma}, Y\rangle}{|\dot{\gamma}||Y|}=\frac{b(s)}{\sqrt{a(s)^{2}+b(s)^{2}}}=\text { constant } \\
\cos \widehat{\dot{\gamma}, T}=\frac{\langle\dot{\gamma}, T\rangle}{|\dot{\gamma}||T|}=0 \Longrightarrow \widehat{\dot{\gamma}, T}=\pi / 2 .
\end{gathered}
$$
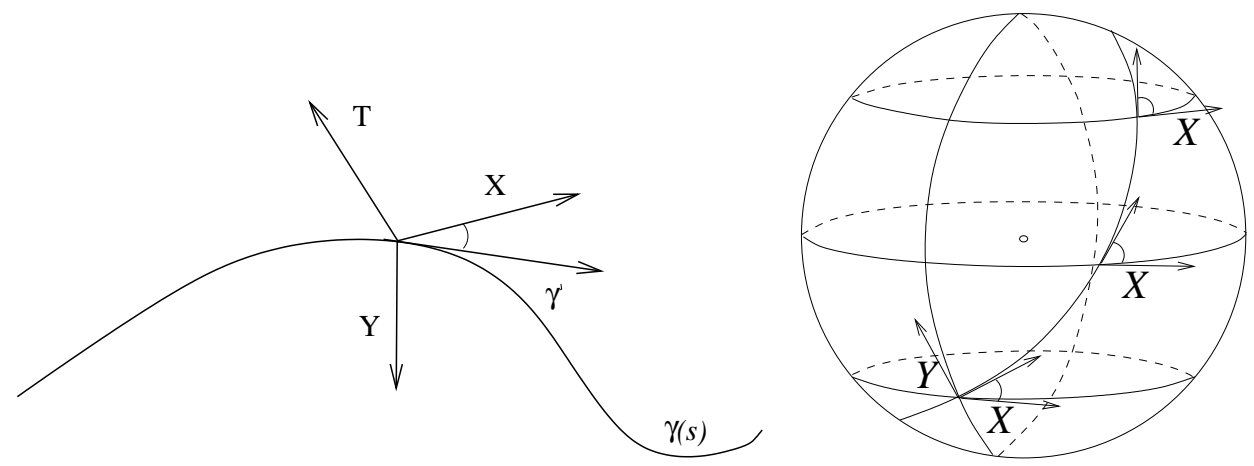

Figure 1: The angle between the velocity $\dot{\gamma}(s)$ and the vectors $X$ and $Y$ is constant.

Remark 5.2 This case when the Lagrange multiplier vanishes yields in the case of the Heisenberg group lines parallel to the $x$-space. These are particular cases of subRiemannian geodesics. 


\subsection{The case $\lambda(s) \neq 0$.}

The Lagrangian in this case is

$$
L=\frac{1}{2} a^{2}+\frac{1}{2} b^{2}+\lambda\left(\dot{x}_{1} y_{1}+\dot{x}_{2} y_{2}-\dot{y}_{1} x_{1}-\dot{y}_{2} x_{2}\right) .
$$

The Euler-Lagrange system (5.15) - (5.16) becomes

$$
\begin{aligned}
& \dot{a} x_{2}+\dot{b} y_{2}=-2\left(a \dot{x}_{2}+b \dot{y}_{2}+\lambda \dot{y}_{1}\right)-\dot{\lambda} y_{1} \\
& \dot{a} x_{1}+\dot{b} y_{1}=-2\left(a \dot{x}_{1}+b \dot{y}_{1}-\lambda \dot{y}_{2}\right)+\dot{\lambda} y_{2} \\
& \dot{a} y_{2}-\dot{b} x_{2}=-2\left(a \dot{y}_{2}-b \dot{x}_{2}+\lambda \dot{x}_{1}\right)-\dot{\lambda} x_{1} \\
& \dot{a} y_{1}-\dot{b} x_{1}=-2\left(a \dot{y}_{1}-b \dot{x}_{1}-\lambda \dot{x}_{2}\right)+\dot{\lambda} x_{2} .
\end{aligned}
$$

Multiplying the first equation by $x_{2}$, the second by $x_{1}$, the third by $y_{2}$ and the forth by $y_{1}$, adding yields

$$
\begin{aligned}
& \dot{a}(\underbrace{x_{1}^{2}+x_{2}^{2}+y_{1}^{2}+y_{2}^{2}}_{=1})+\dot{b}(\underbrace{y_{2} x_{2}+y_{1} x_{1}-x_{2} y_{2}-x_{1} y_{1}}_{=0}) \\
= & -2\left(a(\underbrace{x_{2} \dot{x}_{2}+x_{1} \dot{x}_{1}+y_{2} \dot{y}_{2}+y_{1} \dot{y}_{1}}_{=0})+b(\underbrace{x_{2} \dot{y}_{2}+x_{1} \dot{y}_{1}-y_{2} \dot{x}_{2}-y_{1} \dot{x}_{1}}_{=0})\right. \\
& \left.+\lambda(\underbrace{x_{2} \dot{y}_{1}-x_{1} \dot{y}_{2}+\dot{x}_{1} y_{2}-\dot{x}_{2} y_{1}}_{=b})\right)+\dot{\lambda}(\underbrace{-x_{2} y_{1}+x_{1} y_{2}-x_{1} y_{2}+x_{2} y_{1}}_{=0}) \Longleftrightarrow \\
\dot{a}= & -2 \lambda b .
\end{aligned}
$$

In a similar way, multiplying the first equation by $y_{2}$, the second by $y_{1}$, the third by $-x_{2}$ and the forth by $-x_{1}$, adding we obtain

$$
\begin{aligned}
& \dot{a}(\underbrace{x_{2} y_{2}+x_{1} y_{1}-x_{2} y_{2}-x_{1} y_{1}}_{=0})+\dot{b}(\underbrace{y_{2}^{2}+y_{1}^{2}+x_{2}^{2}+x_{1}^{2}}_{=1}) \\
& =-2\left(a(\underbrace{\dot{x}_{2} y_{2}+\dot{x}_{1} y_{1}-\dot{y}_{2} x_{2}-\dot{y}_{1} x_{1}}_{=0})+b\left(y_{2} \dot{y}_{2}+y_{1} \dot{y}_{1}+x_{2} \dot{x}_{2}+x_{1} \dot{x}_{1}\right)\right. \\
& +\lambda(\underbrace{\dot{y}_{1} y_{2}-\dot{y}_{2} y_{1}-\dot{x}_{1} x_{2}+\dot{x}_{2} x_{1}}_{=-a})+\dot{\lambda}(\underbrace{-y_{1} y_{2}+y_{2} y_{1}-x_{1} x_{2}-x_{2} x_{1}}_{=0}) \Longleftrightarrow \\
& \dot{b}=2 \lambda a \text {. }
\end{aligned}
$$

Hence $a$ and $b$ satisfy the following system

$$
\begin{aligned}
\dot{a} & =-2 \lambda b \\
\dot{b} & =2 \lambda a .
\end{aligned}
$$


Multiplying the first equation by $a$ and the second by $b$ and add, yields $a \dot{a}+b \dot{b}=0$, i.e., $a^{2}+b^{2}=r^{2}$ constant along the geodesics. This means that the energy is preserved and the velocity of the geodesics have constant length. Let $\theta(s)$ be a function such that

$$
a(s)=r \cos \theta(s), \quad b(s)=r \sin \theta(s) .
$$

Substituting in the equations (5.17), (5.18), we obtain

$$
\begin{aligned}
\sin \theta(s) \dot{\theta}(s) & =2 \lambda(s) \sin \theta(s) \\
\cos \theta(s) \dot{\theta}(s) & =2 \lambda(s) \cos \theta(s),
\end{aligned}
$$

which after dividing the first equation by $\sin \theta$ and the second by $\cos \theta$ yields

$$
\dot{\theta}(s)=2 \lambda(s) .
$$

The solution is $\theta(s)=2 \Lambda(s)+\theta_{0}$, where $\Lambda(s)=\int_{0}^{s} \lambda(u) d u$. Hence

$$
\begin{aligned}
& a(s)=r \cos \left(2 \Lambda(s)+\theta_{0}\right), \\
& b(s)=r \sin \left(2 \Lambda(s)+\theta_{0}\right) .
\end{aligned}
$$

Since $\Lambda(0)=0$, then $a(0)=r \cos \theta_{0}$ and $b(0)=r \sin \theta_{0}$, which provides $\theta_{0}=\tan ^{-1}(b(0) / a(0))$.

Let $\gamma(s)$ be a subRiemannian geodesic. Since $\{X, Y\}$ are orthonormal,

$$
a=\langle\dot{\gamma}, X\rangle=\underbrace{|\dot{\gamma}|}_{=r} \cdot \underbrace{|X|}_{=1} \cos (\widehat{\dot{\gamma}, X})=r \cos (\widehat{\dot{\gamma}, X})
$$

and

$$
b=\langle\dot{\gamma}, Y\rangle=\underbrace{|\dot{\gamma}|}_{=r} \cdot \underbrace{|Y|}_{=1} \cos (\widehat{\dot{\gamma}, Y})=r \cos (\widehat{\dot{\gamma}, Y})=r \sin \left(\frac{\pi}{2}-\widehat{\dot{\gamma}, Y}\right) .
$$

Comparing with (5.19), (5.20) we arrive at the following result.

Proposition 5.3 The angles under which the subRiemannian geodesics intersect the integral curves of the vector fields $X$ and $Y$ are given by the formulas

$$
\dot{\gamma}\left(\widehat{s), X_{\gamma(s)}}=2 \Lambda(s)+\theta_{0}, \quad \dot{\gamma}\left(\widehat{s), Y_{\gamma(s)}}=\frac{\pi}{2}-\dot{\gamma}\left(\widehat{s), X_{\gamma}(s)} .\right.\right.\right.
$$

The main goal now is to find $\Lambda(s)$. In order to do this we shall construct an equivalent variational problem. We need the following result, which writes the energy in a more friendly way.

Proposition 5.4 If $\left(x_{1}(s), x_{2}(s), y_{1}(s), y_{2}(s)\right)$ is a subRiemannian geodesic then we have

$$
\dot{x}_{1}^{2}(s)+\dot{x}_{2}^{2}(s)+\dot{y}_{1}^{2}(s)+\dot{y}_{2}^{2}(s)=a^{2}(s)+b^{2}(s) .
$$


Proof: Using the definitions of $a$ and $b$, the horizontality condition, and the holonomic constraint, we can write the following system

$$
\begin{aligned}
& \dot{x}_{1} x_{2}+y_{1} \dot{y}_{2}-\dot{x}_{2} x_{1}-\dot{y}_{1} y_{2}=a(s) \\
& \dot{x}_{1} y_{2}+x_{2} \dot{y}_{1}-\dot{x}_{2} y_{1}-\dot{y}_{2} x_{1}=b(s) \\
& \dot{x}_{1} y_{1}+\dot{x}_{2} y_{2}-x_{2} \dot{y}_{2}-x_{1} \dot{y}_{1}=0 \\
& \dot{x}_{1} x_{1}+\dot{x}_{2} x_{2}+\dot{y}_{1} y_{1}+\dot{y}_{2} y_{2}=0 .
\end{aligned}
$$

This can be written in a matrix way as

$$
\underbrace{\left(\begin{array}{cccc}
x_{2} & -x_{1} & -y_{2} & y_{1} \\
y_{2} & -y_{1} & x_{2} & -x_{1} \\
y_{1} & y_{2} & -x_{1} & -x_{2} \\
x_{1} & x_{2} & y_{1} & y_{2}
\end{array}\right)}_{=M}\left(\begin{array}{l}
\dot{x}_{1} \\
\dot{x}_{2} \\
\dot{y}_{1} \\
\dot{y}_{2}
\end{array}\right)=\left(\begin{array}{l}
a \\
b \\
0 \\
0
\end{array}\right) .
$$

Since $\operatorname{det} M=1, M^{-1}=M^{t}, M$ is an orthogonal matrix, and hence preserves the Euclidean length of vectors. It follows that

$$
\dot{x}_{1}^{2}+\dot{x}_{2}^{2}+\dot{y}_{1}^{2}+\dot{y}_{2}^{2}=a^{2}+b^{2}
$$

along any subRiemannian geodesic, which proves the statement.

Remark 5.5 The above result holds because the Euclidean metric on $\mathbb{R}^{4}$ restricted on $\operatorname{span}\{X, Y\}$ is the subRiemannain metric.

The next result will be useful later in the sequel, when we shall find the Lagrange multiplier.

Proposition 5.6 Let $\gamma(s)$ be a subRiemannain geodesic. Then $\left\langle\ddot{\gamma}(s), T_{\gamma(s)}\right\rangle=0$, for any $s$, i.e., the component of the acceleration along the missing direction $T=[X, Y]$ vanishes.

Proof: Let $\gamma(s)=\left(x_{1}(s), x_{2}(s), y_{1}(s), y_{2}(s)\right)$. Differentiating in the horizontality condition

$$
\dot{x}_{1} y_{1}+\dot{x}_{2} y_{2}-\dot{y}_{1} x_{1}-\dot{y}_{2} x_{2}=0
$$

yields $\ddot{x}_{1} y_{1}+\ddot{x}_{2} y_{2}-\ddot{y}_{1} x_{1}-\ddot{y}_{2} x_{2}=0$, which can be written as

$$
\left\langle\left(\ddot{x}_{1}, \ddot{x}_{2}, \ddot{y}_{1}, \ddot{y}_{2}\right),\left(y_{1}, y_{2},-x_{1},-x_{2}\right)\right\rangle=0,
$$

or $\langle\ddot{\gamma}, T\rangle=0$, where

$$
T=y_{1} \partial_{x_{1}}+y_{2} \partial_{x_{2}}-x_{1} \partial_{y_{1}}-x_{2} \partial_{y_{2}}
$$


We shall consider another variational problem, where we replace the energy $\frac{1}{2}\left(a^{2}+b^{2}\right)$ by $\frac{1}{2}\left(\dot{x}_{1}^{2}+\dot{x}_{2}^{2}+\dot{y}_{1}^{2}+\dot{y}_{2}^{2}\right)$ and consider the new Lagrangian

$$
L^{*}(x, \dot{x}, y, \dot{y})=\frac{1}{2}\left(\dot{x}_{1}^{2}+\dot{x}_{2}^{2}+\dot{y}_{1}^{2}+\dot{y}_{2}^{2}\right)+\lambda(s)\left(\dot{x}_{1} y_{1}+\dot{x}_{2} y_{2}-\dot{y}_{1} x_{1}-\dot{y}_{2} x_{2}\right) .
$$

The Euler-Lagrange equations provided by the Lagrangian $L^{*}$ describe the same subRiemannian geodesics as the equations associated with the initial Lagrangian $L$. The Euler-Lagrange equations for $L^{*}$ are

$$
\begin{aligned}
& \ddot{x}_{1}=2 \lambda \dot{y}_{1}+\dot{\lambda} y_{1} \\
& \ddot{x}_{2}=2 \lambda \dot{y}_{2}+\dot{\lambda} y_{2} \\
& \ddot{y}_{1}=-2 \lambda \dot{x}_{1}-\dot{\lambda} x_{1} \\
& \ddot{y}_{2}=-2 \lambda \dot{x}_{2}-\dot{\lambda} x_{2} .
\end{aligned}
$$

Multiplying the first equation by $y_{1}$, the second by $y_{2}$, the third by $-x_{1}$, and the fourth by $-x_{2}$, adding yields

$$
\begin{aligned}
y_{1} \ddot{x}_{1}+y_{2} \ddot{x}_{2}-x_{1} \ddot{y}_{1}-x_{2} \ddot{y}_{2}= & 2 \lambda(\underbrace{y_{1} \dot{y}_{1}+y_{2} \dot{y}_{2}+x_{1} \dot{x}_{1}+x_{2} \dot{x}_{2}}_{=0}) \\
& +\dot{\lambda}(\underbrace{y_{1}^{2}+y_{2}^{2}+x_{1}^{2}+x_{2}^{2}}_{=1}),
\end{aligned}
$$

which can be written as

$$
\begin{aligned}
\dot{\lambda}(s) & =y_{1} \ddot{x}_{1}+y_{2} \ddot{x}_{2}-x_{1} \ddot{y}_{1}-x_{2} \ddot{y}_{2} \\
& =\left\langle\left(\ddot{x}_{1}, \ddot{x}_{2}, \ddot{y}_{1}, \ddot{y}_{2}\right),\left(y_{1}, y_{2},-x_{1},-x_{2}\right)\right\rangle \\
& =\langle\ddot{\gamma}, T\rangle .
\end{aligned}
$$

Using Proposition 5.6 we obtain the following result.

Proposition 5.7 The Lagrange multiplier $\lambda(s)$ is constant along any subRiemannian geodesics.

If let $\lambda=c / 2$, with $c$ constant, then Proposition 5.3 yields

$$
\dot{\gamma}\left(\widehat{s), X_{\gamma(s)}}=c s+\theta_{0} .\right.
$$

This leads to the following characterization of the subRiemannian geodesics.

Proposition 5.8 The unit speed curve $\gamma(s)$ is a subRiemannian geodesic on $\left(\mathbb{S}^{3}, X, Y\right)$ if and only if the angle between its velocity and the direction of the vector field $X$ increases linearly in $s$. 


\subsection{The group $S U(2)$}

The special unitary group

$$
S U(2)=\left\{x_{1}+x_{2} \mathbf{i}+y_{1} \mathbf{j}+y_{2} \mathbf{k} ; \quad x_{1}^{2}+x_{2}^{2}+y_{1}^{2}+y_{2}^{2}=1\right\}
$$

is also a Lie group, which can be identified with the sphere $\mathbb{S}^{3}$ by the isomorphism

$$
\varphi: S U(2) \rightarrow \mathbb{S}^{3}, \quad \varphi\left(x_{1} I+x_{2} \mathbf{i}+y_{1} \mathbf{j}+y_{2} \mathbf{k}\right)=\left(x_{1}, x_{2}, y_{1}, y_{2}\right) .
$$

Since

$$
\begin{aligned}
X_{q} & =x_{2} \partial_{x_{1}}-x_{1} \partial_{x_{2}}-y_{2} \partial_{y_{1}}+y_{1} \partial_{y_{2}} \\
& =\left(x_{2},-x_{1},-y_{2}, y_{1}\right) \cdot \nabla_{q}=\left(x_{2}-x_{1} \mathbf{i}-y_{2} \mathbf{j}+y_{1} \mathbf{k}\right) \cdot \nabla_{q} \\
& =-\left(x_{1} I+x_{2} \mathbf{i}+y_{1} \mathbf{j}+y_{2} \mathbf{k}\right) \mathbf{i} \cdot \nabla_{q}=-\left(x_{1}, x_{2}, y_{1}, y_{2}\right) \mathbf{i} \cdot \nabla_{q} \\
& =-\left\langle(q \mathbf{i}), \nabla_{q}\right\rangle \\
Y_{q} & =y_{2} \partial_{x_{1}}-y_{1} \partial_{x_{2}}+x_{2} \partial_{y_{1}}-x_{1} \partial_{y_{2}} \\
& =\left(y_{2},-y_{1}, x_{2},-x_{1}\right) \cdot \nabla_{q}=\left(y_{2} I-y_{1} \mathbf{i}+x_{2} \mathbf{j}-x_{1} \mathbf{k}\right) \cdot \nabla_{q} \\
& =-\left(x_{1} I+x_{2} \mathbf{i}+y_{1} \mathbf{j}+y_{2} \mathbf{k}\right) \mathbf{k} \cdot \nabla_{q}=-\left(x_{1}, x_{2}, y_{1}, y_{2}\right) \mathbf{k} \cdot \nabla_{q} \\
& =-\left\langle(q \mathbf{k}), \nabla_{q}\right\rangle, \\
T_{q} & =y_{1} \partial_{x_{1}}+y_{2} \partial_{x_{2}}-x_{1} \partial_{y_{1}}-x_{2} \partial_{y_{2}} \\
& =\left(y_{1}, y_{2},-x_{1},-x_{2}\right) \cdot \nabla_{q}=\left(y_{1} I+y_{2} \mathbf{i}-x_{1} \mathbf{j}-x_{2} \mathbf{k}\right) \cdot \nabla_{q} \\
& =-\left(x_{1} I+x_{2} \mathbf{i}+y_{1} \mathbf{j}+y_{2} \mathbf{k}\right) \mathbf{j} \cdot \nabla_{q}=-\left(x_{1}, x_{2}, y_{1}, y_{2}\right) \mathbf{j} \cdot \nabla_{q} \\
& =-\left\langle(q \mathbf{j}), \nabla_{q}\right\rangle
\end{aligned}
$$

where $q=\left(x_{1}, x_{2}, y_{1}, y_{2}\right), \nabla_{q}=\left(\partial_{x_{1}}, \partial_{x_{2}}, \partial_{y_{1}}, \partial_{y_{2}}\right)$ and $\mathbf{i}, \mathbf{j}, \mathbf{k}$ are defined in (2.1). It follows that the left invariant vector fields $X, Y, T$, which span the Lie algebra of $\mathbb{S}^{3}$ correspond on $S U(2)$ to $-\mathbf{i},-\mathbf{k},-\mathbf{j}$, respectively.

Remark. An alternative approach to calculate geodesics is to use the Hamiltonian method. This method is very complicated in the case of studying geodesics on $\mathbb{S}^{3}$, but some information we can obtain from the superficial analysis. Using the notations (5.21), (5.22), (5.23), the vector fields $X, Y$ and $T$ can be written in the form

$$
X=\left\langle-q I_{1}, \nabla_{q}\right\rangle, \quad Y=\left\langle-q I_{3}, \nabla_{q}\right\rangle, \quad T=\left\langle-q I_{2}, \nabla_{q}\right\rangle
$$

where $\langle\cdot, \cdot\rangle$ is the usual scalar product in $\mathbb{R}^{4}$. Define the Hamilton function

$$
H=\frac{1}{2}\left(X^{2}+Y^{2}\right)=\frac{1}{2}\left(\left\langle q I_{1}, \xi\right\rangle^{2}+\left\langle q I_{3}, \xi\right\rangle^{2}\right) .
$$


Then the Hamilton system is the following

$$
\begin{aligned}
& \dot{q}=\frac{\partial H}{\partial \xi} \Rightarrow \dot{q}=\left\langle q I_{1}, \xi\right\rangle \cdot\left(q I_{1}\right)+\left\langle q I_{3}, \xi\right\rangle \cdot\left(q I_{3}\right) \\
& \dot{\xi}=-\frac{\partial H}{\partial q} \Rightarrow \dot{\xi}=\left\langle q I_{1}, \xi\right\rangle \cdot\left(\xi I_{1}\right)+\left\langle q I_{3}, \xi\right\rangle \cdot\left(\xi I_{3}\right) .
\end{aligned}
$$

A geodesic is the projection of the solution of Hamilton's system to the $q$-space. Since $\left\langle q I_{1}, q\right\rangle=$ $\left\langle q I_{2}, q\right\rangle=\left\langle q I_{3}, q\right\rangle=0$, multiplying the first equation of (5.24) by $q$ we get

$$
\langle\dot{q}, q\rangle=0 \Rightarrow|q|^{2}=\text { const } .
$$

We conclude that the solution of the Hamiltonian system belongs to a sphere. Taking the constant equals 1 we get geodesics belonging to $\mathbb{S}^{3}$. Multiplying the first equation of (5.24) by $q I_{2}$, we get

$$
\left\langle\dot{q}, q I_{2}\right\rangle=0,
$$

by the role of multiplication between $I_{1}, I_{2}$, and $I_{3}$. The reader easily recognize the horizontality condition $\langle\dot{x}, y\rangle=\langle x, \dot{y}\rangle$ in (5.25). It means that the solution of the Hamiltonian system is a horizontal curve. Multiplying the first equation of (5.24) by $q I_{1}$ or by $q I_{3}$, we get

$$
\left\langle\xi, q I_{1}\right\rangle=\left\langle\dot{q}, q I_{1} \mathbf{i}\right\rangle, \quad\left\langle\xi, q I_{3}\right\rangle=\left\langle\dot{q}, q I_{3}\right\rangle .
$$

We see that the Hamilton function can also be written in the form

$$
H=\frac{1}{2}\left(\left\langle q I_{1}, \xi\right\rangle^{2}+\left\langle q I_{3}, \xi\right\rangle^{2}\right)=\frac{1}{2}\left(\left\langle q I_{1}, \dot{q}\right\rangle^{2}+\left\langle q I_{3}, \dot{q}\right\rangle^{2}\right) .
$$

If we multiply the first equation of $(5.24)$ by $\dot{q}$ then we get

$$
|\dot{q}|^{2}=\left\langle q I_{1}, \xi\right\rangle^{2}+\left\langle q I_{3}, \xi\right\rangle^{2}=\left\langle q I_{1}, \dot{q}\right\rangle^{2}+\left\langle q I_{3}, \dot{q}\right\rangle^{2}=2 H .
$$

Thus the Hamiltonian function give the kinetic energy $H=\frac{|\dot{q}|^{2}}{2}$ and it is a constant along the geodesics. Notice that $\left\langle q I_{1}, \dot{q}\right\rangle=a$ and $\left\langle q I_{3}, \dot{q}\right\rangle=b$ by (3.4) and (3.5). We conclude $|\dot{q}|^{2}=a^{2}+b^{2}$ from (5.26) that corresponds to Proposition 5.4. This is only the beginning of a long term project. There are many problems remain open. For example, given any two points on $\mathbb{S}^{3}$, how many geodesics connecting them? Is there any abnormal minimizer in this case? What is the action function? What is the volume element which is the solution of a transport equation? We will answer these questions in a forthcoming paper.

\section{References}

[1] R. Beals, B. Gaveau and P.C. Greiner: Hamilton-Jacobi theory and the heat kernel on Heisenberg groups, J. Math. Pures Appl., 79, \#7 (2000), 633-689. 
[2] R. Beals, B. Gaveau and P.C. Greiner: Complex Hamiltonian mechanics and parametrices for subelliptic Laplacians, I, II, III, Bull. Sci. Math., 21 (1997), 1-36, 97-149, $195-259$.

[3] R. Beals, B. Gaveau and P.C. Greiner: On a geometric formula for the fundamental solution of subelliptic Laplacians, Math. Nachr., 181 (1996), 81-163.

[4] R. Beals and P.C. Greiner: Calculus on Heisenberg manifolds, Ann. Math. Studies \#119, Princeton University Press, Princeton, New Jersey, 1988.

[5] O. Calin, D.C. Chang and P.C. Greiner: Geometric mechanics on the Heisenberg group, Bull. Inst. Math., Academia Sinica, 33, \#3 (2005), 185-252.

[6] O. Calin, D.C. Chang and P.C. Greiner: Heisenberg group and Its Generalizations, AMS/IP series in Advanced Math., International Press, Cambridge, Massachusetts, to appear in 2007.

[7] D.C. Chang and I. Markina: Geometric analysis on quaternion H-type groups, J. Geometric Analysis, 16, \#2 (2006), 265-294.

[8] W.L. Chow: Uber Systeme von linearen partiellen Differentialgleichungen erster Ordnung, Math. Ann., 117 (1939), 98-105.

[9] B. Gaveau: Principe de moindre action, propagation de la chaleur et estimées souselliptiques sur certains groupes nilpotent, Acta Math., 139 (1977), 95-153.

[10] R. Strichartz: Sub-Riemannian geometry, J. Diff. Geometry, 24 (1986), 221-263.

Department of Mathematics, Eastern Michigan University, Ypsilanti, MI, 48197, USA

e-mail: ocalin@emunix.emich.edu

Department of Mathematics, Georgetown University, Washington D.C., 20057, USA

e-mail: chang@math.georgetown.edu

Department of Mathematics, University of Bergen, Johannes Brunsgate 12, Bergen 5008, Nor-

way

e-mail: irina.markina@uib.no 